\title{
Ecstasy-induced oxidative stress to adolescent rat brain mitochondria in vivo: influence of monoamine oxidase type A
}

\author{
Ema Alves ${ }^{1,5}$, Teresa Summavielle ${ }^{1,2}$, Cecília Juliana Alves', José Barata Antunes Custódio ${ }^{3}$, \\ Eduarda Fernandes ${ }^{4}$, Maria de Lourdes Bastos ${ }^{5}$, Maria Amélia Tavares ${ }^{1,6}$ \& Félix Carvalho ${ }^{5}$ \\ Grupo Neurocomportamento, Instituto de Biologia Molecular e Celular (IBMC), University of Porto (UP), Porto, Portugal', Departamento de Ciências Biomédicas, \\ Escola Superior de Tecnologia da Saúde, Instituto Politécnico do Porto, Porto, Portugal2, Biochemistry Department, Faculty of Pharmacy, University of Coimbra, \\ Coimbra, Portugal ${ }^{3}$, REQUIMTE, Physical-Chemistry Department, Faculty of Pharmacy ${ }^{4}$, REQUIMTE, Toxicology Department, Faculty of Pharmacy ${ }^{5}$ and Institute of \\ Anatomy, Medical School of Porto, UP, Porto, Portugal ${ }^{6}$
}

\begin{abstract}
The administration of a neurotoxic dose of 3,4-methylenedioxymethamphetamine (MDMA; 'ecstasy') to the rat results in mitochondrial oxidative damage in the central nervous system, namely lipid and protein oxidation and mitochondrial DNA deletions with subsequent impairment of the correspondent protein expression. Although these toxic effects were shown to be prevented by monoamine oxidase B inhibition, the role of monoamine oxidase A (MAO-A) in MDMA-mediated mitochondrial damage remains to be evaluated. Thus, the aim of the present study was to clarify the potential interference of a specific inhibition of MAO-A by clorgyline, on the deleterious effects produced by a binge administration of a neurotoxic dose of MDMA (10 mg MDMA $/ \mathrm{kg}$ of body weight, intraperitoneally, every 2 hours in a total of four administrations) to an adolescent rat model. The parameters evaluated were mitochondrial lipid peroxidation, protein carbonylation and expression of the respiratory chain protein subunits II of reduced nicotinamide adenine dinucleotide dehydrogenase (NDII) and I of cytochrome oxidase (COXI). Considering that hyperthermia has been shown to contribute to the neurotoxic effects of MDMA, another objective of the present study was to evaluate the body temperature changes mediated by MDMA with a MAO-A selective inhibition by clorgyline. The obtained results demonstrated that the administration of a neurotoxic binge dose of MDMA to an adolescent rat model previously treated with the specific MAO-A inhibitor, clorgyline, resulted in synergistic effects on serotonin- (5-HT) mediated behaviour and body temperature, provoking high mortality. Inhibition of MAO-A by clorgyline administration had no protective effect on MDMA-induced alterations on brain mitochondria (increased lipid peroxidation, protein carbonylation and decrease in the expression of the respiratory chain subunits NDII and COXI), although it aggravated MDMA-induced decrease in the expression of COXI. These results reinforce the notion that the concomitant use of MAO-A inhibitors and MDMA is counter indicated because of the resulting severe synergic toxicity.
\end{abstract}

Keywords 3,4-methylenedioxymethamphetamine, brain mitochondria, hyperthermia, monoamine oxidase A, neurotoxicity, oxidative stress.

Correspondence to: Félix Carvalho, REQUIMTE, Toxicology Department, Faculty of Pharmacy, University of Porto, Rua Anibal Cunha, 164, 4099-030 Porto, Portugal. E-mail: felixdc@ff.up.pt and Teresa Summavielle, Instituto de Biologia Molecular e Celular (IBMC), Rua do Campo Alegre 823, 4150-180 Porto, Portugal. E-mail: TSummavi@ibmc.up.pt.

\section{INTRODUCTION}

The widespread consumption of 3,4-methylenedioxymethamphetamine (MDMA; 'ecstasy'), especially among adolescents and young adults, is presently a matter of high concern because of the acute and chronic toxic effects mediated by this drug. Among the toxic effects mediated by MDMA, its potential to induce damage to the serotonergic nerve terminals is considered a soaring risk because of its irreversible nature. It may result in permanent neurological deficits like sleep disorders, depressed mood, persistent elevation of anxiety, impulsiveness, hostility and selective impairment of episodic memory, working memory and attention (Morgan 2000; Morton 2005).

Several factors may contribute to the MDMA-induced neurotoxicity, namely MDMA metabolism, sustained receptor stimulation, hyperthermia, enzymatic and 
non-enzymatic oxidation of neurotransmitters, inhibition of neurotransmitters synthesis, inflammation and oxidative stress. In addition, we have recently given a new insight on the neurotoxicity of MDMA by showing that, in the rat, the administration of a neurotoxic dose results in monoamine oxidase B (MAO-B)-dependent mitochondrial oxidative damage in the central nervous system (CNS), namely lipid and protein oxidation and mitochondrial DNA (mtDNA) deletion, with subsequent impairment in the corresponding protein expression (Alves et al. 2007). The corresponding mechanism involves MDMAmediated release of neurotransmitters [mainly serotonin (5-HT), but also noradrenaline and dopamine] from storage vesicles and their oxidative deamination by MAO-B, with the consequent production of hydrogen peroxide $\left(\mathrm{H}_{2} \mathrm{O}_{2}\right) . \mathrm{H}_{2} \mathrm{O}_{2}$ may be converted into the highly reactive hydroxyl radical (HO), leading to $\mathrm{HO}$ - mediated oxidative stress damage (Alves et al. 2007). Because MAO enzymes are located in the outer membrane of the mitochondria (Zhuang, Hogan \& McCauley 1988; Zhuang, Marks \& McCauley 1992), $\mathrm{H}_{2} \mathrm{O}_{2}$ easily diffuses through mitochondrial membranes, leading to an oxidative damage of mitochondrial macromolecules, namely lipids, proteins and DNA (Alves et al. 2007). This study corroborated previous findings, showing that MDMAinduced chronic 5-HT loss requires the activity of MAO-B and involves oxidative stress (Schmidt 1987; Sprague \& Nichols 1995; Sprague, Everman \& Nichols 1998). Noteworthy, the strong neuroprotective effect of MAO-B inhibition was independent of body temperature (Alves et al. 2007).

In the CNS, two isoforms of MAO have been described: MAO-A and MAO-B. MAO-A is expressed predominantly in catecholaminergic neurons, whereas MAO-B is expressed in serotonergic neurons, astrocytes and glia (Shih, Grimsby \& Chen 1999), which implies that the metabolism of monoamine neurotransmitters inside serotonergic nerve terminals is mediated by MAO-B, and explains the protective effect of MAO-B inhibitors against MDMA-mediated neurotoxicity. Nevertheless, it has also been demonstrated that the selective inhibition of MAO-A also results in a significant increase of extraneuronal 5-HT in some areas of the rat brain after administration of MDMA (Hewton, Salem \& Irvine 2007; Stanley, Salem \& Irvine 2007). Considering that the post-synaptic effect mediated by acute MAO-A inhibition is physiologically significant under MDMA use, it may be important to evaluate the putative protective effects of MAO-A selective inhibitors against the MDMA-induced neurotoxicity at the mitochondrial level.

Thus, the aim of the present study was to evaluate the interference of a specific inhibition of MAO-A, by clorgyline, on the deleterious effects produced by a binge administration of a neurotoxic dose of MDMA [10 mg
MDMA/kg of body weight, intraperitoneally (i.p.), every 2 hours in a total of 4 administrations] in brain mitochondria of adolescent male Wistar rats. The parameters evaluated were lipid peroxidation, protein carbonylation and expression of the respiratory chain protein subunits II of reduced nicotinamide adenine dinucleotide dehydrogenase (NDII) and I of cytochrome c oxidase (COXI). Considering that hyperthermia has been shown to contribute to the neurotoxic effects of MDMA both in vivo (Malberg \& Seiden 1998) and in vitro (Capela et al. 2006a,b), another objective of the present study was to evaluate the effects of a selective inhibition of MAO-A, by clorgyline, on the MDMA-induced hyperthermia.

\section{MATERIALS AND METHODS}

\section{Animal model}

Wistar rats born from nulliparous females purchased from Charles River Laboratories España S.A. (Barcelona, Spain) were used. Institutional guidelines were followed for animal care. Animals were kept under controlled environmental conditions (temperature, $20 \pm 2{ }^{\circ} \mathrm{C}$; relative humidity, 45-55\%; 12 hours, light/dark cycle) and housed with food and water supplied ad libitum.

On post-natal day 40 (PND 40), two male Wistar rats were caged together. Two days later, a subcutaneous probe (Implantable Programmable Temperature Transponder, IPTT'т-200; Plexx, AB Elst, the Netherlands) (Kort et al. 1998) was inserted in the lumbar region to allow the measurement of body temperature throughout the experiment.

Animals were divided into four experimental groups: MDMA group, clorgyline + MDMA group, clorgyline control group and an isovolumetric saline $(0.9 \% \mathrm{NaCl})$ control group. On PND 45, the animals assigned to the MDMA group received a freshly prepared solution of $10 \mathrm{mg}$ MDMA/kg of body weight, i.p. every 2 hours, in a total of four administrations. The schedule dose was chosen to mimic the common 'binge' pattern of recreational MDMA use, which can be experimentally simulated in animals using repeated drug administration in a single day (Sanchez et al. 2004). The clorgyline + MDMA group received the same dose: 30 minutes was preceded by a single administration of $1 \mathrm{mg} / \mathrm{kg}$ of clorgyline ( $\mathrm{HCl}$ salt; Sigma, Steinheim, Germany). Control animals received an equal dose of saline vehicle $(0.9 \% \mathrm{w} / \mathrm{v})$ in the same protocol of administration and the clorgyline group was administrated a single dose of clorgyline $(1 \mathrm{mg} / \mathrm{kg})$. Body temperature was measured before the first MDMA or isovolumetric saline injection and every 15 minutes for a period of 9 hours. Humane end-points were clearly defined to avoid unnecessary pain and distress of the experimental animals. Signs that would predict imminent death were used to decide the use of timely euthanasia 
(Toth 2000). MDMA (HCl salt) was extracted and purified from high purity MDMA tablets that were provided by the Portuguese Criminal Police Department. The obtained salt was pure and fully characterized by nuclear magnetic resonance and mass spectrometry methodologies.

\section{Mitochondria isolation}

Fourteen days after exposure (PND 59), the animals were killed by decapitation. The encephalon was rapidly removed and processed for brain mitochondria isolation. Mitochondria were isolated from a whole brain by slight modification of a previously described method (Rosenthal et al. 1987). Briefly, the whole encephalon was washed, minced and homogenized at $4^{\circ} \mathrm{C}$ in isolation medium [250 mM sucrose, $5 \mathrm{mM}$ Hepes, $1 \mathrm{mM}$ ethylene glycolbis (2-aminoethyl)-N,N,N',N'-tetraacetic acid (EGTA), $1 \mathrm{mg} / \mathrm{ml}$ bovine serum albumine (BSA), $\mathrm{pH}=7.4$ ] containing $5 \mathrm{mg}$ of bacterial protease (P-5380; Sigma, St. Louis, MO). Single brain homogenates were brought to $30 \mathrm{ml}$ and centrifuged at $1260 \times \mathrm{g}$ (SORVAL RC 5B Plus; Kendro Laboratory Products, Newtown, CT) for 5 minutes. The pellet was discarded and the supernatant was centrifuged at $20000 \times \mathrm{g}$ for 10 minutes. The pellet, including the fluffy synaptosomal layer, was re-suspended in $10 \mathrm{ml}$ of isolation medium containing $0.02 \%$ digitonin (to release mitochondria from the synaptosomal fraction) and centrifuged at $20000 \times \mathrm{g}$ for 10 minutes. The brown mitochondrial pellet (without the synaptosomal layer) was re-suspended in $10 \mathrm{ml}$ of washing medium $(250 \mathrm{mM}$ sucrose, $5 \mathrm{mM}$ Hepes, $\mathrm{pH}=7.4$ ) and centrifuged at $20000 \times \mathrm{g}$ for 10 minutes. The main portion of the mitochondrial pellet was re-suspended in $300 \mu \mathrm{l}$ of washing medium. Mitochondrial protein was determined by the biuret method calibrated with BSA (Gornall, Bardawill \& David 1949).

\section{Quantification of lipid peroxides}

Lipid peroxidation was determined by measuring malondialdehyde (MDA) equivalents, using the thiobarbituric acid (TBA) assay, according to a modified procedure described by Rohn, Hinds \& Vincenzi 1993. Mitochondrial protein $(3 \mathrm{mg})$ was incubated for 30 minutes at $25^{\circ} \mathrm{C}$ in $3 \mathrm{ml}$ of medium [175 mM KCL, $10 \mathrm{mM}$ Tris, $(\mathrm{pH}=7.4$ ) and $3 \mu \mathrm{M}$ rotenone]. Aliquots of $0.3 \mathrm{ml}$ were then incubated with $2.7 \mathrm{ml}$ of thiobarbituric acid reactive substances (TBARS) reagent [9\% TBA, $0.6 \mathrm{~N}$ hydrochloric acid $(\mathrm{HCl})$ and $0.0056 \%$ butylated hydroxyl toluene]. The mixture was warmed to $80-90^{\circ} \mathrm{C}$, for 15 minutes and cooled by immersion in ice for 10 minutes, before centrifuged at $1500 \mathrm{~g}$ for 5 minutes. Lipid peroxidation was estimated by spectrophotometric determination at $535 \mathrm{~nm}$ of the MDA equivalents produced. The amount of MDA equivalents formed was calculated using a molar extinction coefficient of $1.56 \times 10^{5} / \mathrm{mol} / \mathrm{cm}$ and expressed as nmol MDA equivalents/mg protein (Buege \& Aust 1978).

\section{Quantification of protein carbonyls}

Protein carbonyls were quantified by reaction with 2,4dinitrophenylhydrazine (DNPH), with an adapted spectrophotometric method for carbonyl assay (Reznick \& Packer 1994). Two $1 \mathrm{ml}$ aliquots of each mitochondrial extract of $1 \mathrm{mg} / \mathrm{ml}$ were placed in glass tubes. To one tube, $4 \mathrm{ml}$ of $10 \mathrm{mMDNPH}$ in $2.5 \mathrm{M} \mathrm{HCl}$ solution was added, and to the other only $4 \mathrm{ml}$ of $2.5 \mathrm{M} \mathrm{HCl}$ (blank tube). The tubes were left for 1 hour at room temperature in the dark and vortexed every 15 minutes. At this point, $5 \mathrm{ml}$ of $20 \%$ trichloroacetic acid (TCA; w/v) solution was added to both DNPH and $\mathrm{HCl}$ samples to a final concentration of $10 \%$ $(\mathrm{w} / \mathrm{v})$ TCA. The tubes were left in ice for 10 minutes and then centrifuged at $2300 \times \mathrm{g}$ for 5 minutes. The resultant supernatant was discarded. Another wash was subsequently performed with $4 \mathrm{ml}$ of $10 \% \mathrm{TCA}$, and the protein pellets were broken mechanically. The protein pellets were washed three times with ethanol-ethyl acetate $(1: 1 \mathrm{v} / \mathrm{v})$. The final pellet was dissolved in $2 \mathrm{ml}$ of $6 \mathrm{M}$ guanidine hydrochloride solution and left for 10 minutes at $37^{\circ} \mathrm{C}$ in agitation in a water bath. All samples were then centrifuged to remove any insoluble material remaining in suspension. The concentration of DNPH was determined at $360 \mathrm{~nm}$ and a molar absorption coefficient of $22 \times 10^{3} /$ $\mathrm{M} / \mathrm{cm}$ was used to quantify the levels of protein carbonyls. Protein concentration was calculated by determining the absorbance at $280 \mathrm{~nm}$. Protein carbonyl content was expressed as nmol carbonyl formed/mg of mitochondrial protein (Reznick \& Packer 1994).

\section{Western blot analysis of isolated whole brain mitochondria}

Isolated whole brain mitochondria were re-suspended in extraction buffer [20 mM Tris-HCl, pH 7.6, $250 \mathrm{mM}$ sucrose, $40 \mathrm{mM} \mathrm{KCl}, 2 \mathrm{mM}$ ethylenedioxybis (ethyleneamino) tetraacetic acid (EGTA)]. The homogenate was centrifuged at $600 \times \mathrm{g}$ for 10 minutes at $4^{\circ} \mathrm{C}$ and the supernatant was taken for mitochondrial western blot analysis; $15 \mu \mathrm{g}$ of protein were loaded per lane and separated on $10 \%$ sodium dodecyl sulfate-polyacrylamide gels. The gels were transferred to a polyvinylidene fluoride membrane for protein blotting $(0.2 \mu \mathrm{m}$; Bio-Rad Laboratories, Hercules, CA) membranes by electroblotting for 1 hour at $150 \mathrm{~mA}$. The filters were blocked in 5\% non-fat dry milk and $0.1 \%$ Tween 20 overnight at $4^{\circ} \mathrm{C}$. Blots were then incubated with mouse monoclonal antibody against complex IV subunit I (Invitrogen, Eugene, OR; $2 \mu \mathrm{g} / \mathrm{ml}$ ) or complex I subunit II (Invitrogen; $0.5 \mu \mathrm{g} / \mathrm{ml}$ ) diluted in Tris-Buffered Saline Tween 20 (TBST) (0.1\% TBST; $20 \mathrm{mM}$ Tris, $137 \mathrm{mM} \mathrm{NaCl}$, pH 7.6) for 1 hour at room 


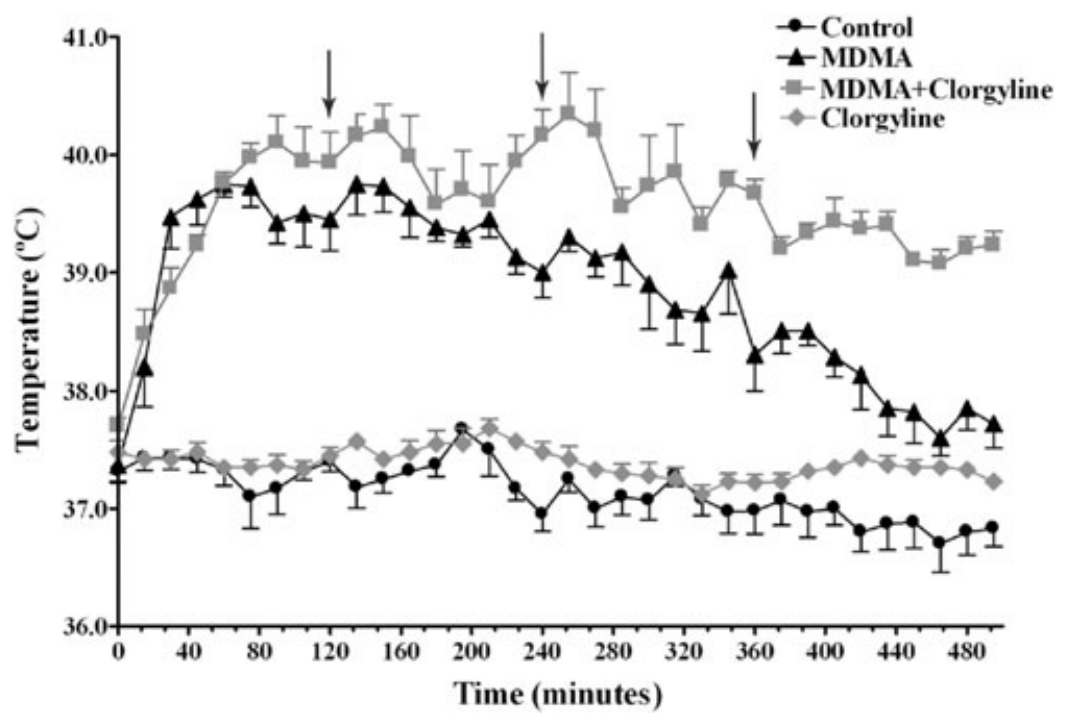

Figure I 3,4-methylenedioxymethamphetamine (MDMA) induced hyperthermia in adolescent male Wistar rats. The image represents body temperature evolution (measured by scanning an inserted subcutaneously probe every 15 minutes for 9 hours) throughout the period of exposure to MDMA $(4 \times 10 \mathrm{mg} / \mathrm{kg})$, clorgyline $(\mathrm{I} \mathrm{mg} / \mathrm{kg})+$ MDMA $(4 \times 10 \mathrm{mg} / \mathrm{kg})$, control saline (isovolumetric saline) and clorgyline (I mg/kg). Clorgyline was administered 30 minutes before exposure to MDMA. Results are reported as mean \pm standard error of the mean for $n=6$ in all groups except clorgyline +MDMA, where $n=3$. Evolution curves for MDMA and MDMA + clorgyline exposed rats started to display significantly higher values than the curves of control and selegiline exposed rats 30 minutes after the first dose $(P<0.00$ I for most time points, two-way analysis of variance followed by a post hoc Tukey Honestly Significant Difference for unequal $n$ ). Arrows indicate injection timings

temperature. Membranes were washed three times for 10 minutes in the same buffer and incubated for 1 hour with horseradish peroxidase (HRP)-conjugated goat anti-mouse IgG (Immun-Star ${ }^{\mathrm{TM}}$; Bio-Rad; 1:20 000). Immuno-reactive proteins were revealed using enhanced chemiluminescence methodology (Immun-Star ${ }^{\mathrm{TM}}$ HRP Chemiluminescent Kit). Blots were analyzed with Quantity One ${ }^{\circledR}$ Software, version 4.5. (Bio-Rad).

\section{Statistical analysis}

Data concerning evolution of body temperature were analysed using a two-way analysis of variance (ANOVA; treatment versus time). Significant differences were further tested using the post hoc Tukey Honestly Significant Difference for unequal $n$.

Data concerning MDA equivalents, protein carbonyls and western blots quantifications were analysed using an appropriated non-parametric alternative to the one-way independent-samples ANOVA, the Kruskal-Wallis test. The statistical level of significance was considered at $P<0.05$. All analyses were performed using SPSS 12.0.0 (SPSS, Inc., Chicago, IL).

\section{RESULTS}

Effects of MDMA + clorgyline administration on body temperature and survival

On the day of exposure (PND 45), rats treated with MDMA $(4 \times 10 \mathrm{mg} / \mathrm{kg}, n=6)$ and MDMA + clorgyline
( $1 \mathrm{mg} / \mathrm{kg}, 30$ minutes before MDMA treatment, $n=3$ ) showed significantly higher body temperatures when compared with saline controls-(isovolumetric $0.9 \% \mathrm{NaCl}$ solution, $n=6)$ and clorgyline- $(1 \mathrm{mg} / \mathrm{kg}, n=6)$ administered animals (Fig. 1). The clorgyline control group did not show significant differences in body temperature when compared with the saline control group (Fig. 1). After the third injection, body temperature started to decline for the MDMA group and attained the control values about 7.5 hours after the first administration, while for clorgyline + MDMA-treated animals, the hyperthermic effect was maintained fairly high (Fig. 1). Importantly, about $57 \%$ of the animals injected with clorgyline + MDMA (four out of seven rats) died or were euthanized before the last administration of MDMA as the pre-defined humane end points were met. The death of these animals was preceded by 'serotonin syndrome' (SS) characteristic behaviour features, namely head weaving, forepaw treading, tremor, flat body posture and backward movement. Noradrenergic dysfunction was also observed by increased secretion of fluids through nose and mouth (increased salivation). Noteworthy, the body temperature of these animals - just before deceasing — was around $41^{\circ} \mathrm{C}$, well above those that survived.

\section{Effects of MDMA + clorgyline administration on lipid peroxidation}

Lipid peroxidation was estimated on whole brain mitochondrial homogenates, 14 days after exposure. The 


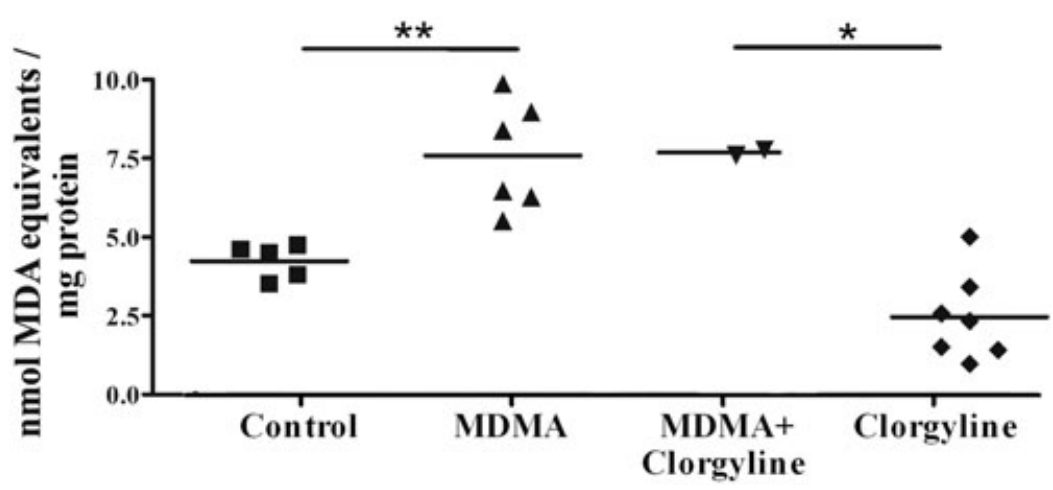

Figure 2 3,4-methylenedioxymethamphetamine (MDMA) induced lipid peroxidation in whole brain mitochondria. Lipid peroxidation was determined by measuring malondialdehyde (MDA) equivalents using the thiobarbituric acid (TBA) assay. Animals were killed 14 days after exposure to MDMA $(4 \times 10 \mathrm{mg} / \mathrm{kg})$, clorgyline $(1 \mathrm{mg} / \mathrm{kg})+$ MDMA $(4 \times 10 \mathrm{mg} / \mathrm{kg})$, saline (isovolumetric saline) or clorgyline (I mg/kg). Clorgyline was administered 30 minutes before exposure to MDMA. Individual values are shown for each experimental group, expressed in nmol MDA equivalents/mg protein, ( $n=5$ for control saline, $n=6$ for MDMA, $n=2$ for MDMA + clorgyline and $n=7$ for clorgyline). Average values for each group are represented by horizontal bars. Significant differences between groups are signed as $* P<0.05$ and $* * P<0.0$ I as evaluated by the non parametric test Kruskal-Wallis

levels of MDA equivalents were measured on the four groups under study: MDMA (40 mg/kg, $n=6)$, MDMA + clorgyline $\quad(40 \mathrm{mg} / \mathrm{kg}+1 \mathrm{mg} / \mathrm{kg}, \quad n=2), \quad$ clorgyline $(1 \mathrm{mg} / \mathrm{kg}, n=7)$ and isovolumetric saline $(0.9 \% \mathrm{NaCl}$, $n=5)$. MDMA-exposed rats displayed significantly higher levels of MDA equivalents than those administered with isovolumetric saline $(P<0.01)$. Rats administered with clorgyline before exposure to MDMA also presented significant higher levels of MDA equivalents than rats that were solely administered with clorgyline $(P<0.05)$. No significant differences were detected between MDMA and MDMA + clorgyline, or between the control saline and clorgyline groups (Fig. 2).

\section{Effects of MDMA + clorgyline administration on the formation of protein carbonyls}

Protein carbonyls were measured on whole brain mitochondrial homogenates of the four groups under study: MDMA (40 mg/kg, $n=7$ ), MDMA + clorgyline (40 mg/ $\mathrm{kg}+1 \mathrm{mg} / \mathrm{kg}, n=2)$, clorgyline $(1 \mathrm{mg} / \mathrm{kg}, n=6)$ and isovolumetric saline $(0.9 \% \mathrm{NaCl}, n=6), 14$ days after exposure. Protein carbonylation was significantly higher in MDMA $(P<0.05)$ and MDMA + clorgyline $(P<0.01)$ groups than in both control groups. The MDMA + clorgyline group also presented higher protein carbonylation than the clorgyline group $(P<0.05)$. Significantly different values were not detected between control saline and clorgyline groups (Fig. 3).

\section{Western blot analysis for the expression of mitochondrial subunit NDII}

The expression of the NDII (complex I of the mitochondrial respiratory chain) was measured on whole brain mitochondrial homogenates, 14 days after exposure; NDII suffered a significant decrease $(P<0.05)$ on its expression after exposure to a neurotoxic dose of MDMA ( $40 \mathrm{mg} / \mathrm{kg}, n=6$ ). A pre-administration of clorgyline did not produce any significant alteration on the pattern of expression of this mitochondrial subunit when compared with MDMA-treated animals. The results for both controls isovolumetric saline $(0.9 \% \mathrm{NaCl}, n=6)$ and clorgyline $(1 \mathrm{mg} / \mathrm{kg}, n=6)$ were not different at the tested level (Fig. 4).

\section{Western blot analysis for the expression of mitochondrial subunit COXI}

The expression of the COXI (complex IV of mitochondrial respiratory chain) was measured on whole brain mitochondrial homogenates, 14 days after exposure.

The expression of COXI was significantly reduced $(P<0.01)$ on the MDMA group, $(40 \mathrm{mg} / \mathrm{kg}, n=6) \mathrm{com}-$ paratively to the control group $(0.9 \% \mathrm{NaCl}, n=6)$. The administration of clorgyline before MDMA (1 mg/kg, $n=2)$ produced a significant decrease $(P<0.05)$ on the expression of this mitochondrial subunit compared with MDMA-treated animals and clorgyline-administered animals $(P<0.05)$. No differences were observed between isovolumetric saline and clorgyline groups (Fig. 5).

\section{DISCUSSION}

The results obtained in the present study demonstrate that the selective inhibition of MAO-A by clorgyline has no beneficial influence on the MDMA-mediated oxidative damage in whole brain mitochondria of rats in vivo. In fact, although the administration of a neurotoxic binge 


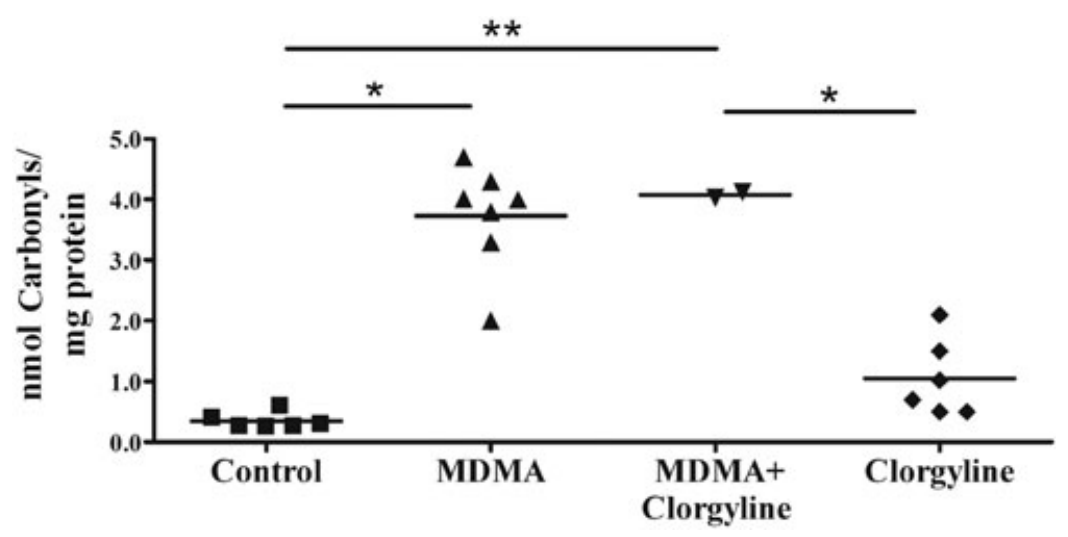

Figure 3 3,4-methylenedioxymethamphetamine (MDMA) induced carbonyl formation in whole brain. Protein carbonyls were quantified by reaction with 2,4-dinitrophenylhydrazine using a spectrophotometric assay for carbonyls. Animals were killed I 4 days after exposure to MDMA $(4 \times 10 \mathrm{mg} / \mathrm{kg})$, clorgyline $(\mathrm{I} \mathrm{mg} / \mathrm{kg})+$ MDMA $(4 \times 10 \mathrm{mg} / \mathrm{kg})$, saline (isovolumetric saline) or clorgyline $(\mathrm{I} \mathrm{mg} / \mathrm{kg})$. Clorgyline was administered 30 minutes before exposure to MDMA. Individual values are shown for each experimental group expressed in nmol carbonyls/mg protein, ( $n=6$ for control saline, $n=7$ for MDMA, $n=2$ for MDMA + clorgyline and $n=6$ for clorgyline). Average values for each group are represented by horizontal bars. Significant differences between groups are signed as $* P<0.05$ and $* * P<0.0$ I as evaluated by the non-parametric test Kruskal-Wallis

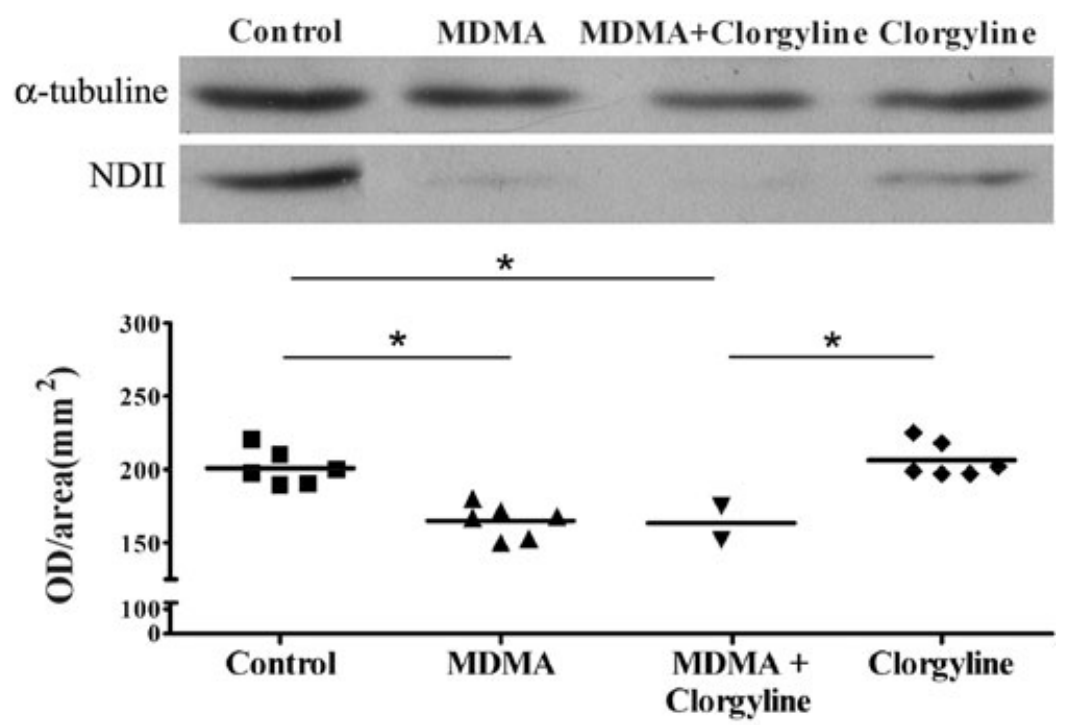

Figure 4 3,4-methylenedioxymethamphetamine (MDMA) induced decreased expression of the subunit II of reduced nicotinamide adenine dinucleotide dehydrogenase (NDII) in whole brain mitochondria. NDII expression was determined by western blot analysis performed with isolated whole brain mitochondria of male Wistar rats, using a mouse monoclonal antibody anti-NDII subunit. Animals were killed I4 days after exposure to MDMA $(4 \times 10 \mathrm{mg} / \mathrm{kg})$, clorgyline $(I \mathrm{mg} / \mathrm{kg})+$ MDMA $(4 \times 10 \mathrm{mg} / \mathrm{kg})$, saline (isovolumetric saline) or clorgyline $(I \mathrm{mg} / \mathrm{kg})$. Clorgyline was administered 30 minutes before exposure to MDMA. Individual values are shown for each experimental group expressed in optical density/area $\left(\mathrm{mm}^{2}\right), n=6$ for all groups except for MDMA + clorgyline $(n=2)$. Average values for each group are represented by horizontal bars. Densitometric analysis of $\alpha$-tubulin served as loading control. Significant differences in the expression of NDII between groups are signed as $* P<0.05$ and $* P<0.01$ as evaluated by the non-parametric test Kruskal-Wallis

dose of MDMA (10 mg MDMA/kg of body weight, i.p., every 2 hours in a total of four administrations) resulted in significant lipid peroxidation, protein carbonylation and decreased expression of the respiratory chain proteins NDII and COXI; the inhibition of MAO-A by clorgyline $(1 \mathrm{mg} / \mathrm{kg}), 30$ minutes before MDMA administration, had no protective effect on the modified parameters. On the contrary, it was observed that MAO-A inhibition resulted in about $57 \%$ mortality during the course of MDMA administration. Importantly, the death of these animals was preceded by an increased SS characteristic behavioural features and signs of adrenergic dysfunction.

Also of note, the hyperthermic effect on the clorgyline + MDMA-treated animals was maintained 

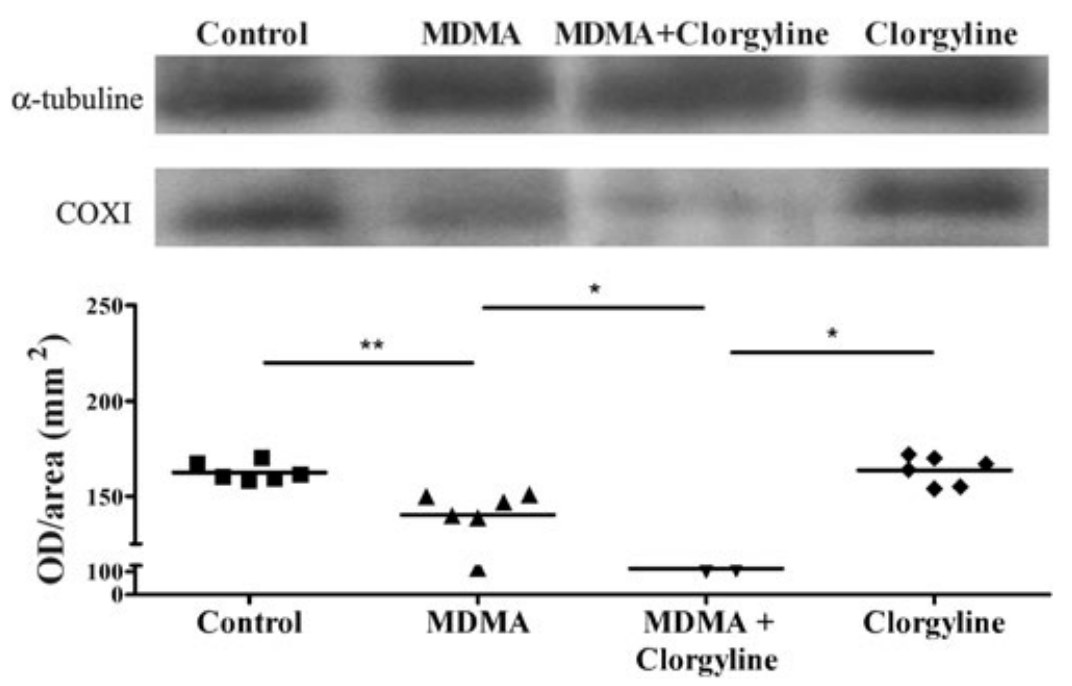

Figure 5 3,4-methylenedioxymethamphetamine (MDMA) induced decreased expression of the subunit I of cytochrome oxidase (COXI) in whole brain mitochondria. COXI expression was determined by western blot analysis performed with isolated whole brain mitochondria of male Wistar rats, using a mouse monoclonal antibody anti-COXI subunit. Animals were killed I 4 days after exposure to MDMA $(4 \times 10 \mathrm{mg} / \mathrm{kg})$, clorgyline $(2 \mathrm{mg} / \mathrm{kg})+$ MDMA $(4 \times 10 \mathrm{mg} / \mathrm{kg})$, saline (isovolumetric saline) or clorgyline $(2 \mathrm{mg} / \mathrm{kg})$. Clorgyline was administered 30 minutes before exposure to MDMA. Individual values are shown for each experimental group expressed in optical density/area $\left(\mathrm{mm}^{2}\right), n=6$ for all groups except for MDMA + clorgyline $(n=2)$. Average values for each group are represented by horizontal bars. Densitometric analysis of $\alpha$-tubulin served as loading control. Significant differences in the expression of subunits II of reduced nicotinamide adenine dinucleotide dehydrogenase between groups are signed as $* P<0.05$ and $* * P<0.01$ as evaluated by the non-parametric test Kruskal-Wallis

fairly stable during the whole time of MDMA administration, although in the MDMA group it started to decline after the third administration. Furthermore, the body temperature of these animals, just before decreasing, was around $41^{\circ} \mathrm{C}$, well above those that survived in both MDMA-exposed groups. One of the first physiological phenomena observed after MDMA exposure is the increase in body temperature (Carvalho et al. 2002; Alves et al. 2007). Our results, showing that the sustained hyperthermic effect observed in animals administered with clorgyline and MDMA that was associated with high lethality, confirm previous studies where MAO-A inhibition together with MDMA exposure resulted in higher temperatures and increased toxicity of the drug associated with a higher rate of mortality (Huether, Zhou \& Ruther 1997).

Our group has recently demonstrated that the exposure of an adolescent rat model to a neurotoxic 'binge' administration of MDMA, induces not only an oxidative stress status in mitochondria, but also a deletion of mtDNA with the consequent impairment of the genes required for particular protein synthesis, which were shown to be essential complexes of the mitochondrial respiratory system required for energy production (Alves et al. 2007). Although deletion of mtDNA was not measured in the present study because of the high mortality observed for the MDMA + clorgyline group, the decrease in the expression of the respiratory chain proteins NDII and COXI in the MDMA and MDMA + clorgyline groups, much probably results from the deletion of a portion of the mitochondrial genome that includes codons for these proteins (Suliman et al. 2002; Suliman, Carraway \& Piantadosi 2003). The pre-treatment with clorgyline potentiated the MDMA-induced decreased expression of COXI, compared with MDMA-treated animals. Thus, although MAO-A inhibition had no effect on MDMAinduced oxidative stress in mitochondria, it potentiated MDMA-induced mitochondrial damage.

Notwithstanding the protection afforded by MAO-B inhibition reported in previous studies against MDMAinduced neurotoxicity, it has long been known that the use of MAO inhibitors may result in a steady increase of synaptic serotonin levels mediated by MDMA (Parrott 2002; Silins, Copeland \& Dillon 2007), This aggravates the SS often observed in ecstasy users, which ultimately may be fatal (Silins et al. 2007). In rodents, the signs of SS include head weaving, forepaw treading, hind limb abduction, dorsiflexion of the tail (straub tail), tremor, flat body posture and backward movement (Izumi et al. 2007), which was corroborated in the present study. In previous studies, including our own, the observed moderate MDMA-induced SS effect was not affected by MAO-B inhibition. Thus, it is possible that the beneficial effects of specific MAO-B inhibitors overwhelm the deleterious effects of the SS. It is also possible that the inhibition of the MAO-A isoform is the one mainly responsible for the high risk reported for the concomitant use of ecstasy with MAO inhibitors such as phenelezine and 
tranylcypromine, nialamide, isoniazid, moclobemide and clorgyline (Vuori et al. 2003; Silins et al. 2007). Avoiding contact with MDMA or other amphetamines is thus a strong reminder to anyone undergoing antidepressant therapy with MAO-A inhibitory drugs, including the abovementioned (for review see Youdim et al., 2006) or to those under treatment with drugs that compete for MAO-A metabolism like Tripans (Armstrong \& Cozza 2002). MAO inhibitors are also present in tobacco leafs (Khalil et al. 2000) and recent positron emission tomography imaging studies have demonstrated a significant decrease in both MAO-A and MAO-B activities in the brains of smokers (Fowler et al. 1996a,b). The influence of this effect on MDMA neurotoxicity is yet to be studied. Furthermore, several plant extracts have been showing to inhibit MAO-A, (Kong et al. 2004; Tomić et al. 2005; Chimenti et al. 2006; Han et al. 2007), which may represent another hazard to those consuming MDMA. Corroborating this rationale in the present study other than the high mortality and potentiation of MDMA-induced mitochondrial damage, it was also observed that MAO-A inhibition led to the aggravation of MDMA-induced behavioural effects, characteristic of both serotonergic (head weaving, forepaw treading, tremor, flat body posture, and backward movement) and over-stimulated adrenergic signs such as of piloerection and increased salivation (Spanos \& Yamamoto 1989).

It has first been suggested, by Sprague and Nichols, that the neurotoxicity induced by MDMA may be explained with the following sequence of events: depletion of 5-HT from serotonergic neurons; increase in dopamine synthesis and release, in part attributable to the stimulation of $5-\mathrm{HT}_{2 \mathrm{~A}}$ receptors; increase in extracellular dopamine; transport of dopamine into 5-HT nerve terminals by the 5-HT transporter; and deamination of dopamine inside 5-HT nerve terminals by MAO-B, with the consequent formation of $\mathrm{H}_{2} \mathrm{O}_{2}$ and related oxidative stress, leading to the selective 5-HT neuronal degeneration (Sprague \& Nichols 1995). Taking into account that MAO-A is expressed predominantly in catecholaminergic neurons (Shih et al. 1999), it may be considered that its inhibition will increase the availability of dopamine for an uptake by serotonergic neurons and subsequent MAO-B metabolism. This hypothesis may explain the lack of protective effects mediated by MAO-A inhibition against MDMA-induced neurotoxicity.

In conclusion, the obtained results demonstrated that the administration of a neurotoxic binge dose of MDMA to an adolescent rat model previously treated with the specific MAO-A inhibitor, clorgyline, resulted in synergistic effects on 5-HT-mediated behaviour, body temperature and provokes high mortality. Also, inhibition of MAO-A had no protective effect on the MDMA-mediated oxidative damage in whole brain mitochondria of these rats, although it aggravated MDMA-induced decrease in the expression of COXI. These results reinforce the notion that the concomitant use of MAO-A inhibitors and MDMA is counter-indicated because of the resulting severe synergic toxicity.

\section{Acknowledgements}

This work was granted by Fundação Calouste Gulbenkian (Principal Investigator: Teresa Summavielle) and 'Programa de Financiamento Plurianual do IBMC'. Ema Alves, Teresa Summavielle and Cecilia Alves were granted by Fundação para a Ciência e Tecnologia (FCT) (SFRH/BD/12176/2003, SFRH/BPD/20997/ 2004, SFRH/BD/17195/2004).

\section{References}

Alves E, Summavielle T, Alves CJ, Gomes-da-Silva J, Barata JC, Fernandes E, Bastos Mde L, Tavares MA, Carvalho F (2007) Monoamine oxidase-b mediates ecstasy-induced neurotoxic effects to adolescent rat brain mitochondria. J Neurosci 27:10203-10210.

Armstrong SC, Cozza KL (2002) Triptans. Psychosomatics 43:502-504.

Buege JA, Aust SD (1978) Microsomal lipid peroxidation. Methods Enzymol 52:302-310.

Capela JP, Meisel A, Abreu AR, Branco PS, Ferreira LM, Lobo AM, Remiao F, Bastos ML, Carvalho F (2006a) Neurotoxicity of Ecstasy metabolites in rat cortical neurons, and influence of hyperthermia. J Pharmacol Exp Ther 316:53-61.

Capela JP, Ruscher K, Lautenschlager M, Freyer D, Dirnagl U, Gaio AR, Bastos ML, Meisel A, Carvalho F (2006b) Ecstasyinduced cell death in cortical neuronal cultures is serotonin 2A-receptor-dependent and potentiated under hyperthermia. Neuroscience 139:1069-1081.

Carvalho M, Carvalho F, Remiao F, de Lourdes Pereira M, Pires-das-Neves R, de Lourdes Bastos M (2002) Effect of 3,4-methylenedioxymethamphetamine ('ecstasy') on body temperature and liver antioxidant status in mice: influence of ambient temperature. Arch Toxicol 76:166-172.

Chimenti F, Cottiglia F, Bonsignore L, Casu L, Casu M, Floris C, Secci D, Bolasco A, Chimenti P, Granese A, Befani O, Turini P, Alcaro S, Ortuso F, Trombetta G, Loizzo A, Guarino I (2006) Quercetin as the active principle of Hypericum hircinum exerts a selective inhibitory activity against MAO-A: extraction, biological analysis, and computational study. J Nat Prod 69:945-949.

Fowler JS, Volkow ND, Wang GJ, Pappas N, Logan J, MacGregor R, Alexoff D, Shea C, Schlyer D, Wolf AP (1996b) Inhibition of monoamine oxidase B in the brains of smokers. Nature 379:733-736.

Fowler JS, Volkow ND, Wang GJ, Pappas N, Logan J, Shea C, Alexoff D, MacGregor R, Schlyer D, Zezulkova I, Wolf AP (1996a) Brain monoamine oxidase A inhibition in cigarette smokers. Proc Natl Acad Sci USA 93:14065-14069.

Gornall AG, Bardawill CJ, David MM (1949) Determination of serum proteins by means of the biuret reaction. J Biol Chem 177:751-766.

Han XH, Hong SS, Hwang JS, Lee MK, Hwang BY, Ro JS (2007) Monoamine oxidase inhibitory components from Cayratia japonica. Arch Pharm Res 30:13-17. 
Hewton R, Salem A, Irvine RJ (2007) Potentiation of 3,4methylenedioxymethamphetamine-induced 5-HT release in the rat substantia nigra by clorgyline, a monoamine oxidase $\mathrm{A}$ inhibitor. Clin Exp Pharmacol Physiol 34:1051-1057.

Huether G, Zhou D, Ruther E (1997) Causes and consequences of the loss of serotonergic presynapses elicited by the consumption of 3,4-methylenedioxymethamphetamine (MDMA, 'ecstasy') and its congeners. J Neural Transm 104:771794.

Izumi T, Iwamoto N, Kitaichi Y, Kato A, Inoue T, Koyama T (2007) Effects of co-administration of antidepressants and monoamine oxidase inhibitors on 5-HT-related behavior in rats. Eur J Pharmacol 565:105-112.

Khalil AA, Steyn S, Castagnoli N Jr (2000) Isolation and characterization of a monoamine oxidase inhibitor from tobacco leaves. Chem Res Toxicol 13:31-35.

Kong LD, Cheng CH, Tan RX (2004) Inhibition of MAO A and B by some plant-derived alkaloids, phenols and anthraquinones. J Ethnopharmacol 91:351-355.

Kort WJ, Hekking-Weijma JM, TenKate MT, Sorm V, VanStrik R (1998) A microchip implant system as a method to determine body temperature of terminally ill rats and mice. Lab Anim 32:260-269.

Malberg JE, Seiden LS (1998) Small changes in ambient temperature cause large changes in 3,4methylenedioxymethamphetamine (MDMA)-induced serotonin neurotoxicity and core body temperature in the rat. J Neurosci 18:5086-5094.

Morgan MJ (2000) Ecstasy (MDMA): a review of its possible persistent psychological effects. Psychopharmacology (Berl) 152:230-248.

Morton J (2005) Ecstasy: pharmacology and neurotoxicity. Curr Opin Pharmacol 5:79-86.

Parrott AC (2002) Recreational Ecstasy/MDMA, the serotonin syndrome and serotonergic neurotoxicity. Pharmacol Biochem Behav 71:837-844.

Reznick AZ, Packer L (1994) Oxidative damage to proteins: spectrophotometric method for carbonyl assay. Methods Enzymol 233:357-363.

Rohn TT, Hinds TR, Vincenzi FF (1993) Ion transport ATPases as targets for free radical damage. Protection by an aminosteroid of the $\mathrm{Ca} 2+$ pump ATPase and $\mathrm{Na}+\mathrm{K}+$ pump ATPase of human red blood cell membranes. Biochem Pharmacol 46:525-534.

Rosenthal RE, Hamud F, Fiskum G, Varghese PJ, Sharpe S (1987) Cerebral ischemia and reperfusion: prevention of brain mitochondrial injury by lidoflazine. J Cereb Blood Flow Metab 7:752-758.

Sanchez V, O'Shea E, Saadat KS, Elliott JM, Colado MI, Green AR (2004) Effect of repeated ('binge') dosing of MDMA to rats housed at normal and high temperature on neurotoxic damage to cerebral 5-HT and dopamine neurones. J Psychopharmacol 18:412-416.
Schmidt CJ (1987) Neurotoxicity of the psychedelic amphetamine, methylenedioxymethamphetamine. J Pharmacol Exp Ther 240:1-7.

Shih JC, Grimsby J, Chen K (1999) Molecular biology of monoamine oxidase $\mathrm{A}$ and $\mathrm{B}$ : their role in the degradation of serotonin. In: HBaM G, ed. Serotoninergic Neurons and 5-HT Receptors in the SNC, pp. 655-670. Berlin: Springer.

Silins E, Copeland J, Dillon P (2007) Qualitative review of serotonin syndrome, ecstasy (MDMA) and the use of other serotonergic substances: hierarchy of risk. Aust N Z J Psychiatry 41:649-655.

Spanos LJ, Yamamoto BK (1989) Acute and subchronic effects of methylenedioxymethamphetamine [(+/-) MDMA] on locomotion and serotonin syndrome behavior in the rat. Pharmacol Biochem Behav 32: 835-840.

Sprague JE, Everman SL, Nichols DE (1998) An integrated hypothesis for the serotonergic axonal loss induced by 3,4methylenedioxymethamphetamine. Neurotoxicology 19: 427-441.

Sprague JE, Nichols DE (1995) The monoamine oxidase-B inhibitor L-deprenyl protects against 3,4-methylenedioxymethamphetamine-induced lipid peroxidation and long-term serotonergic deficits. J Pharmacol Exp Ther 273:667-673.

Stanley N, Salem A, Irvine RJ (2007) The effects of co-administration of 3,4-methylenedioxymethamphetamine ('ecstasy') or para-methoxyamphetamine and moclobemide at elevated ambient temperatures on striatal 5-HT, body temperature and behavior in rats. Neuroscience 146:321-329.

Suliman HB, Carraway MS, Piantadosi CA (2003) Postlipopolysaccharide oxidative damage of mitochondrial DNA. Am J Respir Crit Care Med 167:570-579.

Suliman HB, Carraway MS, Velsor LW, Day BJ, Ghio AJ, Piantadosi CA (2002) Rapid mtDNA deletion by oxidants in rat liver mitochondria after hemin exposure. Free Radic Biol Med 32:246-256

Tomić M, Tovilović G, Butorović B, Krstić D, Janković T, Aljancić I, Menković N (2005) Neuropharmacological evaluation of diethylether extract and xanthones of Gentiana kochiana. Pharmacol Biochem Behav 81:535-542.

Toth LA (2000) Defining the moribund condition as an experimental endpoint for animal research. ILAR J 41:72-79.

Vuori E, Henry JA, Ojanpera I, Nieminen R, Savolainen T, Wahlsten P, Jantti M (2003) Death following ingestion of MDMA (ecstasy) and moclobemide. Addiction 98:365-368.

Youdim MB, Edmondson D, Tipton KF (2006) The therapeutic potential of monoamine oxidase inhibitors. Nat Rev Neurosci 7:295-309.

Zhuang Z, Hogan M, McCauley R (1988) The in vitro insertion of monoamine oxidase B into mitochondrial outer membranes. FEBS Lett 238:185-190.

Zhuang ZP, Marks B, McCauley RB (1992) The insertion of monoamine oxidase A into the outer membrane of rat liver mitochondria. J Biol Chem 267:591-596. 